\title{
Tratamento da endometriose
}

\author{
Treatment of endometriosis
}

Paula Andrea de Albuquerque Salles Navarro ${ }^{1}$, Ionara Diniz Santos Barcelos ${ }^{2}$, Júlio César Rosa e Silva ${ }^{3}$

\section{RESUMO}

Apesar da grande heterogeneidade das manifestações clínicas relacionadas à endometriose, observa-se a sua elevada prevalência em mulheres inférteis e portadoras de dor pélvica crônica. O impacto sócio-econômico desta afecção enigmática é elevado e os dados relacionados à eficácia das diferentes abordagens terapêuticas descritas são bastante conflitantes. Assim, os objetivos do presente artigo são apresentar as evidências científicas disponíveis acerca das diferentes modalidades terapêuticas aplicáveis e estabelecer recomendações para o tratamento da infertilidade e da dor pélvica crônica relacionadas à endometriose. Em pacientes com endometriose mínima ou leve a supressão da função ovariana não é efetiva para melhorar a fertilidade, mas a ablação das lesões associadas à adesiólise parece ser mais efetiva do que a realização exclusiva da laparoscopia diagnóstica. Não há evidências suficientes para determinar se a excisão cirúrgica em casos de doença moderada ou severa melhoraria as taxas de gestação. A fertilização in vitro parece ser uma abordagem adequada, especialmente nos casos de coexistência de fatores de infertilidade e/ou falha de outras abordagens terapêuticas. Deve-se avaliar a possibilidade de usar agonistas do GnRH por 3 a 6 meses, previamente à realização de fertilização in vitro. Em relação ao alívio da dor, verifica-se que a supressão da função ovariana por 3 a 6 meses em pacientes com doença confirmada laparoscopicamente reduz a dor associada à endometriose. Todas as medicações estudadas parecem apresentar eficácia similar, embora os efeitos adversos e de os custos sejam diferentes. A ablação das lesões endometrióticas reduz a dor associada à endometriose, sendo menos efetiva nos casos de doença mínima. A exérese de endometriomas com diâmetro $>4 \mathrm{~cm}$ parece melhorar a taxa de fecundidade natural e após procedimentos de reprodução assistida, além de reduzir a dor e os riscos de recidiva. Por fim, é importante ressaltar que há muita controvérsia e que as recomendações acima descritas deverão ser revistas à medida que estudos clínicos randomizados, controlados e com casuística adequada gerarem evidências mais concretas e confiáveis.

PALAVRAS-CHAVE: Endometrios/terapia; Infertilidade feminina; Dor pélvica; Reprodução; Técnicas reprodutivas assistidas

\section{ABSTRACT}

Despite a wide heterogeneity of clinical manifestations related to endometriosis, a high prevalence of the disease is observed in infertile women and in those with chronic pelvic pain. This enigmatic condition has a high socioeconomic impact, and the described data regarding efficacy of the therapeutic approaches are quite conflicting. Thus, the purpose of the present study was to describe the available scientific evidence about the applicable therapeutic modalities and to provide recommendations for the treatment of infertility and the chronic pelvic pain related to endometriosis. Although suppression of ovarian function in patients with minimal or mild endometriosis is not effective in improving fertility, ablation of the lesions associated with adhesiolysis seems to be more effective than exclusive diagnostic laparoscopy. There is no sufficient evidence to determine whether surgical excision in cases of moderate or severe disease would improve the pregnancy rates. In vitro fertilization seems to be an adequate approach, especially in cases of coexistence of infertility factors and/or failure of other treatments. The possibility of using GnRH for 3 to 6 months before in vitro fertilization should be considered. Regarding pain relief, suppression of ovarian function for 3 to 6 months in patients with laparoscopically-confirmed disease reduces the pain associated with endometriosis. All studied medication seem to have similar efficacy, differing only in terms of adverse effects and costs. Ablation of endometriotic lesion reduces the pain associated with endometriosis, being less effective in cases of minimal disease. Exeresis of endometriomas with diameter $>4 \mathrm{~cm}$ seems to improve the rate of natural fecundity and the rate for ??? obtained after assisted reproduction procedures, in addition to reducing both pain and recurrence risk. Finally, it is important to emphasize that this subject is much controversial and the recommendations herein described should be revised as randomized controlled clinical trials with adequate casuistic generate more concrete and reliable evidence.

KEYWORDS: Endometriosi/therapy; Infertility, female; Chronic pelvic pain; Reproduction; Reproductive techniques, assisted

1 Professora Adjunta da Área de Saúde da Mulher, Faculdade de Medicina, Universidade Federal de São Carlos - UFSCAR - São Carlos (SP), Brasil, Orientadora do Programa de Pós-Graduação, Faculdade de Medicina, Universidade de São Paulo - USP - Ribeirão Preto (SP), Brasil.

2 Pós-Graduanda, Departamento de Ginecologia e Obstetrícia, Faculdade de Medicina, Universidade de São Paulo - USP - Ribeirão Preto (SP), Brasil.

3 Médico Assistente, Setor de Endoscopia Ginecológica e Dor Pélvica, Departamento de Ginecologia e Obstetrícia, Faculdade de Medicina, Universidade de São Paulo - USP - Ribeirão Preto - (SP), Brasil.

Correspondência: Paula Andrea de Albuquerque Salles Navarro

Avenida Caramuru, 1280 - apto 132 - 14030-000 - Ribeirão Preto-SP - e-mail: paasnavarro@uol.com.br

Recebido em: 2/10/2006

Aceito com modificações em: 24/10/2006

Rev Bras Ginecol Obstet. 2006; 28(10): 612-23. 
Introdução

A endometriose é uma doença que afeta a mulher em idade reprodutiva, sendo caracterizada por implante e crescimento de tecido endometrial (glândulas e/ou estroma) fora da cavidade uterina. Tem sido observada em 5 a $10 \%$ das pacientes submetidas a laparotomias ginecológicas, 20 a $50 \%$ das mulheres com infertilidade ${ }^{1}$ e 60 a $70 \%$ das portadoras de dor pélvica crônica ${ }^{2}$.

Esta afecção pode cursar com uma grande diversidade de manifestações clínicas. Podemos encontrar desde pacientes oligo ou assintomáticas até quadros de dor pélvica intensa, sintomas decorrentes de lesão em órgãos não reprodutivos e infertilidade. Os sintomas associados a esta doença geram repercussão em todos os aspectos na vida de suas portadoras, devendo-se dispensar especial atenção a todas as queixas ${ }^{3}$.

O tratamento deve ser individualizado, considerando sempre os sintomas da paciente e o impacto da doença e do tratamento sobre a qualidade de vida. Uma equipe multidisciplinar especializada deve ser (sempre que possivel) envolvida, na tentativa de fornecer um tratamento capaz de abranger todos os aspectos bio-psico-sociais da paciente.

Para fins didáticos, subeste artigo em tratamento da infertilidade e da dor pélvica crônica associada à endometriose. Apresentaremos uma revisão sistematizada da literatura nos tópicos mais importantes e controversos relacionados com o tratamento dos sintomas associados a esta afecção, procurando analisar os dados de maneira crítica, à luz das evidências apresentadas. Contudo, convém ressaltar que a grande maioria dos dados de evidências que suportam as condutas atuais é conflitante. Possivelmente serão revistos à medida que houver estudos bem delineados, prospectivos, randomizados e controlados, com casuística maior e, conseqüentemente, passiveis de gerar evidências mais concretas e com adequado poder de teste.

\section{Tratamento da infertilidade associada à endometriose}

\subsection{Endometriose e fertilidade}

Em casais normais a fecundidade varia de 0,15 a 0,20 por mês, declinando com a idade ${ }^{4}$. A fecundidade mensal de mulheres inférteis com endometriose é significativamente menor, e varia de 0,02 a $0,1^{5}$. Alguns estudos relatam que 20 a $50 \%$ das mulheres inférteis têm endometriose e 30 a 50\% das mulheres com endometriose são inférteis, sugerindo um possivel papel da endometriose na etiopatogênese da infertilidade.

Nos casos de endometriose moderada e severa, em que geralmente se identifica comprometimento morfológico da anatomia pélvica, é clara a associação entre esta afecção e a infertilidade subseqüente, embora uma demonstração clara de relação causal ainda não tenha sido estabelecida. Nos casos de doença mínima e leve, em que não se observa alteração mecânica no trato reprodutivo, esta associação torna-se ainda mais controversa. Contudo, alguns estudos têm evidenciado menores taxas de fecundidade mesmo neste grupo de pacientes $(0,024$ e 0,019$)$, quando comparadas com mulheres férteis normais $(0,15 \text { a } 0,20)^{6,7}$.

\subsection{Tratamento clinico medicamentoso}

Alguns ensaios clínicos randomizados e controlados (RCT) demonstraram que a administração de danazol, progestágenos ou agonistas do (GnRHa) não foi efetiva no tratamento da infertilidade associada a endometriose mínima ou leve. Assim, a supressão da função ovariana por meio de terapia hormonal para melhorar a fertilidade nos casos de endometriose mínima e leve não é efetiva, retarda a possibilidade de gravidez e não deve ser oferecida como abordagem terapêutica única ${ }^{8}$.

Não há evidência de eficácia da supressão transitória na função ovariana em termos de melhora subseqüente da fecundidade em casos de endometriose moderada ou severa, Portanto, não recomendamos esta conduta como rotina isolada na prática clínica, particularmente quando se optou inicialmente pela conduta expectante. Contudo, alguns estudos sugerem haver aumento nas taxas de gestação se uma supressão ovariana prolongada, com GnRHa, for realizada antes do ciclo de reprodução assistida (RA). Há pelo menos dois estudos caso-controle e um RCT que suportam essa abordagem $^{9-11}$. O RCT avaliou, em todos os estágios da doença, 51 mulheres que receberam tratamento por três meses com análogos de GnRH, comparando-as a um grupo que não recebeu nenhum tratamento antes do ciclo de RA. A taxa de gravidez clínica por ciclo iniciado foi de $80 \%$ no grupo tratado versus $54 \%$ no grupo controle; não houve diferença na dosagem utilizada de gonadotrofina ${ }^{11}$. A principal crítica a esse estudo é que a taxa de gravidez relatada foi muito maior que a média nacional (33\%) para o mesmo ano (como relatado pela ASRM / Society for Assisted Reproductive Technology).

Uma recente revisão da Cochrane sugere que a administração de análogos de GnRH por um período de 3 a 6 meses antes de fertilização in vitro 
(FIV) ou injeção intracitoplasmática de espermatozóide (ICSI) em mulheres com endometriose aumenta em quatro vezes a probabilidade de gravidez clínica $^{12}$. Dados sobre os efeitos potencialmente adversos desta abordagem terapêutica na mãe ou no feto ainda não estão disponiveis. Contudo, é importante ressaltar que além da pequena casuística analisada nesta revisão (165 mulheres), dados sobre estadiamento da doença, presença e tempo de infertilidade, motivo da indicação do procedimento de RA, e idade das pacientes incluídas não foram avaliados separadamente. Assim, a adoção desta conduta deveria ser individualizada e discutida com as pacientes, considerando outros fatores potencialmente determinantes do sucesso nos procedimentos de RA.

\subsection{Tratamento cirúrgico}

Os objetivos principais da cirurgia em pacientes com endometriose são retirar a maior quantidade de tecido possivel e restabelecer a anatomia normal da pelve. O manejo delicado do tecido e a hemostasia meticulosa são fundamentais para evitar a formação de novas aderências e focos endometrióticos ${ }^{13}$.

A ablação de lesões endometrióticas, associada a adesiólise, para melhorar a fertilidade nos casos de endometriose mínima e leve é efetiva se for comparada com a laparoscopia diagnóstica apenas ${ }^{14}$. Esta recomendação está baseada em revisão sistemática e meta-análise de dois ensaios clínicos randomizados controlados similares, embora contraditórios. Nestes trabalhos a eficácia da ablação cirúrgica laparoscópica (mais adesiólise) foi comparada (em termos de melhora da fecundidade) com laparoscopia diagnóstica apenas, em portadoras de endometriose mínima e leve. Contudo, o poder de evidência nos dois estudos pode ser questionado. No estudo italiano ${ }^{7}$, em que não se evidenciou melhora na fecundidade para o grupo tratado cirurgicamente, o número de pacientes incluídas foi bastante pequeno (101 pacientes). No estudo canadense ${ }^{6}$ (maior casuística, porém com apenas 341 mulheres incluidas) houve no grupo tratado uma taxa mensal de fecundidade significantemente maior, se comparada à do grupo controle. Entretanto, não se pode afirmar que as pacientes sabiam ter sido ou não submetidas a tratamento cirúrgico durante a laparoscopia, o que introduz um outro viés na análise dos dados obtidos. Não encontramos evidências de que a taxa de fecundidade após o tratamento cirúrgico tenha sido influenciada pelo método de ablação utilizado, seja eletrocirúrgico ou a laser ${ }^{6}$.

Desta forma, até que novos estudos sejam publicados, se durante a realização de laparosco- pia diagnóstica em paciente infértil for identificada presença de endometriose mínima ou leve, deve-se proceder à ablação cirúrgica das lesões e adesiólise. A conduta a seguir, seja indicação de procedimento de RA ou expectante, deve considerar entre outros, fatores como idade da paciente, tempo de infertilidade, tipo e resultados de tratamentos prévios, presença ou não de fator masculino associado. Por outro lado, é muito questionável indicar cirurgia laparoscópica a pacientes com diagnóstico prévio de endometriose mínima e leve e realizar ablação cirúrgica das lesões e adesiólise para melhorar a fecundidade. Acreditamos que esta conduta deveria ser individualizada e discutida com a paciente apenas nas situações em que, após a análise global do caso, a opção pela conduta expectante pareça viável pela possibilidade de concepção espontânea.

Quanto aos casos de endometriose moderada ou severa, apenas dispomos de estudos observacionais que avaliaram a influência do tratamento cirúrgico nas taxas de gestação. Alguns estudos ${ }^{15-17}$ sugerem haver correlação negativa entre estágio da endometriose e taxa cumulativa de gravidez espontânea após remoção cirúrgica das lesões endometrióticas. Entretanto, só foi encontrada significância estatística em um destes estudos ${ }^{17}$. Convém ressaltar que dados obtidos de diferentes estudos não podem ser comparados facilmente, pois os procedimentos cirúrgicos realizados, a extensão das cirurgias, a habilidade do cirurgião e outras variáveis que determinam o resultado certamente não foram padronizadas ${ }^{18}$.

Não dispomos de estudos clínicos randomizados controlados ou de meta-análises que tenham avaliado se a remoção cirúrgica da endometriose moderada ou severa melhora as taxas de gestação. Portanto, não é possivel afirmar que o tratamento cirúrgico da endometriose moderada ou severa melhora as taxas de gravidez subseqüentes. Assim, a indicação desta abordagem também deveria ser individualizada. A avaliação da indicação cirúrgica deve considerar, entre outros, fatores como idade da paciente e presença de outras causas de infertilidade associadas. Apesar da controvérsia, não recomendamos adiar a indicação de procedimentos de RA a pacientes inférteis com idade $>35$ anos em prol da conduta expectante devido à redução no potencial reprodutivo inerente ao avanço na idade.

\subsection{Endometriomas}

Uma revisão recente da Cochrane, para determinar a técnica cirúrgica mais efetiva no tratamento de endometriomas, ablação ou exérese da cápsula, avaliou dois estudos randomizados sobre o manejo laparoscópico de endometriomas com diâmetro > 
$3 \mathrm{~cm}^{19}$. Dois RCT foram incluídos na análise. Os resultados demonstraram que a exérese laparoscópica da cápsula estava associada à diminuição tanto na taxa de recorrência do endometrioma $\left(\mathrm{OR}=0,41 ; \mathrm{IC}_{95 \%}=0,18-0,93\right)$ como na necessidade de reintervenção $\left(\mathrm{OR}=0,21 ; \mathrm{IC}_{95 \%}=0,05-0,79\right)$, além do aumento na taxa de gravidez espontânea (avaliada 12 a 24 meses após a abordagem cirúrgica) em mulheres com subfertilidade documentada $\left(\mathrm{OR}=5,21 ; \mathrm{IC}_{95 \%}=2,04-13,29\right)^{19}$. Independentemente da técnica cirúrgica utilizada, recomenda-se enviar para análise anatomopatológica parte da cápsula do endometrioma para confirmar o diagnóstico clínico e excluir a presença de malignidade (risco estimado de malignização de $0,7 \%)^{19}$.

Segundo a última publicação da Sociedade Européia de Reprodução Humana e Embriologia $(\mathrm{ESHRE})^{18}$, a exérese laparoscópica de endometriomas com diâmetro $>4 \mathrm{~cm}$ melhora a fertilidade, caracterizada pelo aumento nas taxas de gestação espontânea após a intervenção cirúrgica, se comparada à drenagem e coagulação da pseudocápsula $^{20,21}$, estando ao mesmo tempo associada a um menor risco para recorrência do cisto ${ }^{22}$. Contudo, acreditamos que a adoção de tratamento cirúrgico como abordagem exclusiva para infertilidade associada a endometriose requer individualização do caso, como foi mencionado anteriormente.

Nos casos em que se indica realização de procedimentos de reprodução assistida, permanece controversa a influência do endometrioma nos resultados obtidos. Alguns estudos relatam que a presença de endometrioma não afeta os resultados em ciclos de FIV. Outros reportam redução na resposta a indução da ovulação e do número de embriões a serem transferidos, além de maiores taxas de abortamento. Assim, apesar da controvérsia e da ausência de evidências concretas, na última publicação da ESHRE ${ }^{18}$ recomenda-se exérese laparoscópica de endometriomas com diâmetro $>4 \mathrm{~cm}$ previamente ao início da estimulação ovariana controlada. O objetivo da recomendação seria obter material para diagnóstico anatomopatológico, reduzir o risco de infecção, facilitar o acesso técnico aos folículos durante a captação oocitária e, possivelmente, melhorar a resposta ovariana à administração de gonadotrofinas endógenas. Contudo, esta decisão deve ser individualizada e reconsiderada, particularmente nos casos de pacientes com intervenções cirúrgicas prévias.

Se por um lado as evidências suportam o benefício da exérese da pseudocápsula do endometrioma (em detrimento de sua cauterização) em termos de diminuição na taxa de recorrência e na necessidade de reintervenção, (além do aumento na taxa de gravidez espontânea nos meses subse- qüentes), por outro postula-se um efeito potencial deste procedimento sobre a redução na reserva folicular ovariana ${ }^{23-27}$. Contudo, outros estudos ainda são necessários para esclarecer se o dano está relacionado com o procedimento cirúrgico ou com a presença prévia do cisto $^{28}$. De qualquer modo, este fato deveria ser considerado antes e durante o ato operatório, para determinar a melhor abordagem cirúrgica e procurar minimizar o dano ao parênquima ovariano aparentemente normal.

\subsection{Tratamento pré e pós-operatório}

Não há RCT avaliando o efeito do tratamento hormonal antes da intervenção cirúrgica.

O tratamento com danazol ou agonista de GnRH após a cirurgia não melhora a fertilidade, quando comparado com o tratamento expectante ${ }^{29-31}$. Portanto, nos casos em que se optar pela conduta expectante no pós-operatório, não se recomenda utilizar rotineiramente o tratamento hormonal para melhorar a fecundidade natural.

Contudo, como já foi mencionado, a administração de análogos do GnRH durante 3 a 6 meses antes de FIV ou injeção ICSI em mulheres com endometriose aumenta em quatro vezes a probabilidade de gravidez clínica ${ }^{12}$. Assim, nos casos em que se indicar realização de RA, no pósoperatório pode-se adotar esta conduta, que deve ser individualizada e discutida com as pacientes. Deve-se considerar os efeitos adversos do uso de análogos do $\mathrm{GnRH}$, as possiveis conseqüências de adiar o procedimento, bem como os fatores potencialmente determinantes do sucesso nos procedimentos de RA.

\subsection{Tecnologias de reprodução assistida}

A realização de estimulação ovariana controlada com citrato de clomifeno ou gonadotrofinas, associada a inseminação intra-uterina, melhora a fertilidade em pacientes com endometriose mínima e leve. Sugerimos que esta conduta seja adotada, considerando a idade da paciente (preferencialmente < 35 anos), exclusão de fator masculino severo e de falha em tentativas similares prévias, ressaltando-se as taxas esperadas de fecundidade por ciclo $(0,09 \text { a } 0,13)^{32,33}$.

Não dispomos de grandes RCT que definitivamente tenham demonstrado maior eficácia da FIV com transferência embrionária (FIVETE) sobre a conduta expectante no tratamento da infertilidade associada a endometriose. Todavia, a FIVETE tem sido rotineiramente oferecida a estas pacientes. Segundo o último guideline da ESHRE, é uma abordagem apropriada, especialmente nos 
casos de disfunção tubária, presença de fator masculino associado e/ou falha após outras medidas terapêuticas ${ }^{18}$. Outros fatores como idade feminina avançada, diminuição da reserva folicular ovariana e tempo de infertilidade também deveriam ser considerados quando da definição do tratamento a ser oferecido.

Também há controvérsia sobre um possivel papel deletério da endometriose nos resultados de procedimentos de FIVETE. Uma meta-análise publicada em 2002 evidenciou menores taxas de gravidez obtidas após FIVETE em pacientes com endometriose, quando comparadas a pacientes com infertilidade de causa tubária ${ }^{34}$. Contudo, esta meta-análise incluiu estudos antigos, que utilizaram captação oocitária laparoscópica e protocolos menos efetivos de estimulação ovariana. Por outro lado, alguns grandes bancos de dados, como o da Society for Assisted Reproductive Technology, não evidenciaram este efeito adverso da endometriose sobre as taxas de gestação pós-FIVETE.

Como já foi citado, uma revisão recente da Cochrane sugere que administração de análogos de GnRH durante 3 a 6 meses antes de FIV ou ICSI em mulheres com endometriose aumenta em quatro vezes a probabilidade de gravidez clinica ${ }^{12}$. Contudo, a heterogeneidade dos estudos analisados nesta revisão torna as conclusões questionáveis. Portanto, a adoção desta conduta deve ser individualizada e discutida com as pacientes, considerando outros fatores que podem determinar o sucesso dos procedimentos de reprodução assistida.

\section{Tratamento de endometriose associada a dor pélvica}

\subsection{Tratamento clinico de endometriose associada a dor pélvica}

O uso de terapia medicamentosa para endometriose é baseado no fato de que a endometriose responde a hormônios. Duas condições fisiológicas, gravidez e menopausa, estão freqüentemente associadas à resolução da dor provocada pela endometriose. Os análogos farmacológicos destas condições são os progestágenos e contraceptivos orais combinados, que levam a condições hormonais semelhantes à vista durante a gravidez, e os androgênios e GNRHa, que promovem supressão do estrogênio endógeno.

Segundo as evidências documentadas a seguir, pode-se concluir que o tratamento clínico medicamentoso para dor pélvica associada a endometriose é altamente eficaz, com taxas de sucesso que variam de 80 a $100 \%$ de melhora e um intervalo livre dos sintomas que pode chegar a dois anos. As drogas hormonais investigadas - acetato de medroxiprogesterona (AMP), gestrinona, COC, danazol e GnRHa mostram-se igualmente efetivas no alivio da dor. Contudo, os efeitos adversos apresentados e os custos são diferenciados e devem ser levados em consideração quando da escolha terapêutica ${ }^{18}$.

\subsubsection{Progestágenos e contraceptivos orais} combinados

Embora a progesterona cause alterações secretoras no endométrio durante a fase lútea do ciclo menstrual normal, os progestágenos sintéticos causam atrofia endometrial por diminuição na sintese de receptores de estrogênio. Kirstner $(1958)^{35}$, foi o primeiro a usar esta classe de medicamentos para tratar a endometriose com altas doses de progestágenos, levando a um estado de pseudogravidez.

Embora o AMP seja um dos progestágenos mais utilizados para tratar dor pélvica associada a endometriose, os dados da literatura são controversos. Luciano et al. ${ }^{36}$ mostraram melhora de 88 e $83 \%$ em dismenorréia e dispareunia, respectivamente, de pacientes usuárias de AMP oral (50 mg/dia). Os implantes de endometriose apresentavam atrofia e pseudo-decidualização após biópsia realizada durante laparoscopia diagnóstica posterior ${ }^{36}$. Entretanto, em estudo prospectivo, randomizado e controlado por placebo, outros mostraram que esta medicação não levou a melhora significantemente maior que o placebo no controle de dismenorréia em pacientes com endometriose, depois de 12 semanas de uso oral contínuo $(50 \mathrm{mg} / \mathrm{dia})^{37}$. Além disso, esse tipo de medicação está associado a alta incidência de efeitos colaterais (40\%) como acne, dor localizada e vasodilatação.

O AMP de depósito é uma formulação contraceptiva que tem sido adotada por alguns pesquisadores no tratamento da endometriose. Em um estudo controlado comparativo entre AMP de depósito (150 mg a cada 90 dias) e uso de contraceptivo oral combinado associado ao danazol (50 mg/dia), os autores mostraram que os dois tratamentos foram igualmente e altamente efetivos no controle da dor, principalmente após seis meses de tratamento, atingindo-se o máximo de satisfação das pacientes após doze meses. Porém, promoveram alta incidência de efeitos colaterais, apresentados por cerca de 30\% das usuárias, e não houve avaliação da eficácia desta abordagem após doze meses de tratamento ${ }^{38}$.

Gestrinona, um progestagênio com atividade androgênica, também tem sido utilizada no trata- 
mento de endometriose e quando comparada com o GnRHa parece ter efeito semelhante no controle da dor e na satisfação das pacientes ${ }^{39,40}$. Porém, esta medicação apresenta efeitos colaterais, que muitas vezes não são suportados pelas pacientes, como amenorréia, spotting, acne, hirsutismo, edema e ganho de peso, que pode chegar até a $3 \mathrm{~kg}$ em seis meses de tratamento. A posologia adequada é de 200 a 300 mg/semana, e deve ser sempre seguida pelo parâmetro clínico da amenorréia.

Outros progestágenos orais podem ser usados e já foram testados no tratamento da endometriose, como acetato de ciproterona, desogestrel, acetato de noretindrona, levonorgestrel, didrogesterona. Porém, mais estudos devem ser realizados para definir sua utilização em pacientes com dor pélvica e endometriose ${ }^{41,42}$.

Outras vias de administração de progestágenos têm sido testadas, como implante subdérmico de etonorgestrel e o sistema intra-uterino com liberação lenta de levonorgestrel (SIU-LNG). Sobre o primeiro há apenas um estudo piloto na literatura, mostrando boa resposta e satisfação das pacientes $^{43}$. Alguns estudos na literatura evidenciam boa eficácia do SIU-LNG para alivio da dor associada a endometriose. Vercellini et al. (1999) ${ }^{44}$ e Fedele et al. $(2001)^{45}$ foram os primeiros a testar o SIU-LNG na endometriose, mostrando sua boa efetividade no controle da dor. Em outro estudo prospectivo, randomizado e controlado, demonstrou-se diminuição importante, já no primeiro mês de uso, da dor pélvica crônica secundária à endometriose ${ }^{46}$. Associada a melhora clínica, observou-se também a diminuição nos valores séricos de CA-125, marcador tumoral utilizado na prática clínica para acompanhamento da endometriose ${ }^{47}$. Embora os dados referidos sejam promissores, as casuísticas avaliadas são pequenas, não fornecendo evidência suficiente para que se preconize esta abordagem rotineiramente.

Os COC são a primeira escolha para tratamento clínico em muitos centros. No primeiro estudo controlado em que esta terapêutica foi utilizada e comparada ao uso do GnRHa, demonstrou-se que o uso de COC, embora cíclico (21 dias de hormônio e 7 sem medicação), tem eficácia similar à da administração de GnRHa na redução da dor pélvica associada a endometriose ${ }^{48}$. Embora haja controvérsia, alguns estudos não evidenciaram eficácia do uso pós-operatório de COC na postergação do tempo de recorrência da doença ${ }^{49}$. Por ser um tratamento simples, pouco dispendioso, de fácil manejo e com bons resultados embasados pela literatura, os COC têm sido amplamente utilizados no controle da dor pélvica associada a endometriose. Porém, ainda não está claro o mecanismo pelo qual este regime de tratamento age sobre os focos de endometriose.
É interessante notar e considerar que, em pacientes cujo principal sintoma é dismenorréia, o uso contínuo (i.é., sem pausa) de COC leva a amenorréia, promovendo melhores resultados a curto prazo no controle da dismenorréia e na melhora da qualidade de vida das pacientes ${ }^{50}$.

\subsubsection{Danazol}

O danazol é um androgênio que suprime as gonadotrofinas e age inibindo a ovulação. Seu uso no tratamento da endometriose está descrito desde $1971^{51}$. Vários outros estudos comparando esta medicação com GnRHa, COC e progestágenos mostram uma grande eficácia desta droga no tratamento da endometriose, promovendo grande satisfação das pacientes, melhora dos sintomas apresentados e, conseqüentemente, impacto positivo sobre a qualidade de vida ${ }^{52,53}$. Porém, a incidência de efeitos colaterais é muito alta, atingindo cerca de $85 \%$ das usuárias ${ }^{52}$. Os principais efeitos adversos descritos são: ganho de peso, edema, diminuição no tamanho das mamas, acne, hirsutismo, oleosidade na pele e alterações no timbre da $\mathrm{voz}^{52}$, além da potencial influência negativa sobre o metabolismo dos lipídeos, com elevação dos níveis de LDL-colesterol e colesterol total ${ }^{54}$. A posologia adequada é de $600 \mathrm{mg} /$ dia. Em estudo comparativo entre diferentes doses de danazol $(100,200,400$ e $600 \mathrm{mg} /$ dia) para se determinar a melhor posologia, observou-se que, apesar da maior incidência de efeitos colaterais, a dose de $600 \mathrm{mg} /$ dia é a que apresenta melhores resultados no controle da dismenorréia, provavelmente pela amenorréia induzida ${ }^{55}$.

\subsubsection{Agonistas do GnRH}

São considerados hoje o tratamento padrão contra dor associada a endometriose, devido ao estado de hipoestrogenismo que acarretam. Seu uso na endometriose foi descrito pela primeira vez em 1982, em estudo piloto em que 5 pacientes utilizaram esta medicação por via subcutânea diariamente por um mês, apresentando melhora clínica significativa ${ }^{56}$. Desde então, vários estudos foram realizados, comparando esta classe de drogas com os tratamentos para endometriose já existentes. Houve excelente resposta terapêutica, com melhora significativa na dor pélvica associada a endometriose, e maior tempo para retorno dos sintomas e aparecimento de lesões císticas ovarianas (endometriomas) ${ }^{46,57-59}$. A média de tempo para recidiva dos sintomas de dismenorréia, dispareunia e dor pélvica acíclica é muito variável: entre 6 e 12 meses após o final do tratamento, 
porém geralmente a dor é de menor intensida$\mathrm{de}^{60,61}$. Por isso, recomendamos a utilização de outras formas de tratamento após o término do uso do GnRHa, como COC, progestágenos com diferentes vias de administração ou antiinflamatórios não hormonais.

Contudo, o tempo exato de utilização desta classe de medicamento no tratamento clínico da dor associada a endometriose ainda não está definido na literatura. Alguns autores preconizam seu uso por seis meses, outros por três meses, mas a tendência parece ser de utilizá-los pelo menor tempo necessário. Hornstein et al. ${ }^{62}$ compararam o uso de GnRHa por três e seis meses no tratamento de pacientes com dor pélvica associada a endometriose e constataram não haver diferença entre os dois períodos de tratamento quanto à melhora na dor, tempo para recidiva dos sintomas ou ainda necessidade de tratamento clínico ou cirúrgico posterior.

Outra questão que ainda gera dúvidas é a utilização do GnRHa após a cirurgia para endometriose como adjuvante no tratamento desta enfermidade. Vários estudos foram realizados, porém os dados ainda não são conclusivos. Conquanto alguns autores não mostraram benefícios com sua utilização, não evidenciando melhora nos resultados a longo prazo ${ }^{63}$, outros mostraram que a melhora na dor e a postergação da recorrência desta afecção foram mais significativas após o uso destas medicações ${ }^{59}$. Parece que a tendência é sua utilização, porém por um tempo não superior a seis meses.

O grande inconveniente para o uso do GnRHa no tratamento da dor associada a endometriose certamente é a ocorrência de efeitos adversos secundários ao hipoestrogenismo, sendo os principais, as ondas de calor e o ressecamento vaginal, que podem estar presentes em até 90\% dos $\operatorname{casos}^{64}$. Estes efeitos colaterais não são os únicos; entre os menos freqüentes podemos citar cefaléia, tontura, labilidade emocional, acne, mialgia, edema, redução no volume mamário, ganho de peso, diminuição na libido e insônia ${ }^{64}$. Outro grande problema é a perda de massa óssea decorrente do hipoestrogenismo induzido por seu uso, que pode variar de 3 a $6 \%$ em seis meses de uso $^{65}$ e até $12 \%$ em um ano ${ }^{66}$. Para evitar esta perda óssea e minimizar os efeitos colaterais pode-se utilizar a add-back terapia, que seria a utilização de baixas doses de progestágenos isolados ou associados a estrogênios durante o uso do GnRHa ${ }^{67}$. Todavia, considerações cautelosas devem ser feitas quando da análise da prescrição de GnRHa para mulheres jovens, antes da aquisição do pico de massa óssea.

\subsection{Tratamento cirúrgico da endometriose associada à dor pélvica}

Uma avaliação adequada da eficácia das diferentes técnicas cirúrgicas utilizadas na abordagem da endometriose associada à dor pélvica fica dificultada pelo pequeno número de trabalhos randomizados e controlados existentes.

Dependendo da gravidade da doença encontrada, a prática ideal consiste em realizar o diagnóstico e a exérese cirúrgica dos focos de endometriose no mesmo ato operatório. Assim, recomenda-se obter o consentimento livre e esclarecido no pré-operatório, abrangendo caracterização do procedimento que poderá ser realizado, complicações possiveis e taxas de sucesso. Não há dados concretos que justifiquem a prescrição de tratamento hormonal antes da cirurgia, para melhorar as taxas de sucesso na abordagem cirúrgica ${ }^{18}$.

O tratamento pós-operatório com danazol GnRHa por seis meses, após a cirurgia, reduz a dor associada a endometriose e posterga a recorrência da doença, quando comparado com placebo ou conduta expectante. Contudo, o tratamento pósoperatório com COC parece não ser tão efetivo ${ }^{18}$.

$\mathrm{O}$ tratamento cirúrgico pode ser dividido em duas categorias, conservador ou radical, sendo o conservador o que preserva a fertilidade da paciente e o radical aquele que leva à histerectomia e à salpingooforectomia bilateral. Este é erroneamente considerado como tratamento definitivo para endometriose, pois cursa com taxas de recidiva da dor pélvica crônica em até $10 \%$ dos $\operatorname{casos}^{68}$. Por esta razão, parece impróprio considerá-lo definitivo. Contudo, caso a histerectomia e a salpingooforectomia bilateral tenham sido realizadas em pacientes jovens, recomenda-se prescrever terapia de reposição hormonal, cujo melhor regime ainda não está bem estabelecido. Desta forma, deve-se individualizar a prescrição, considerando a relação risco-benefício da terapia de reposição hormonal para cada caso particular.

\subsubsection{Laparoscopia versus laparotomia}

Hoje, com o desenvolvimento e a tecnologia dos aparelhos laparoscópicos, a eficácia desta técnica na abordagem de pacientes com dor pélvica e endometriose é inquestionável, permitindo uma excelente visão da pelve e, conseqüentemente, das lesões endometrióticas. Não há evidências sólidas de qual via de acesso, laparoscópica ou laparotômica, apresenta melhor eficácia no tratamento da doença e da dor relacionada. 
Vários trabalhos foram realizados buscando comparar as duas técnicas cirúrgicas na abordagem da mulher com dor e endometriose, sendo o objetivo final avaliar o tempo de recorrência da dismenorréia, dispareunia e dor acíclica. Em pacientes com dor pélvica moderada ou severa e com endometriose estádio IV, pela American Society of Reproductive Medicine (ASRM), as taxas de recorrência de dor acíclica, dismenorréia e dispareunia após 24 meses de seguimento foram semelhantes para as duas técnicas ${ }^{69}$. Estes dados foram confirmados por vários outros trabalhos ${ }^{70,71}$. Porém, a laparoscopia apresenta vantagens como menor perda sanguínea, tempo de recuperação pós-cirúrgico mais curto, menor dor pós-operatória e tempo de estada no hospital ${ }^{72}$.

\subsubsection{Tratamento laparoscópico}

A destruição das lesões por coagulação, fulguração ou vaporização ou a exérese das lesões superficiais mostram resultados semelhantes quanto à melhora na dor pélvica associada à endometriose ${ }^{73}$. Em um estudo prospectivo e controlado, demonstrou-se que a destruição das lesões endometrióticas tem efeito benéfico, quando comparada apenas com laparoscopia, sem a destruição das lesões ${ }^{74}$. Resultado semelhante foi encontrado por Abbott et al. $(2004)^{75}$, mostrando que as lesões superficiais devem ser sempre destruídas.

Quanto à abordagem dos endometriomas ovarianos em pacientes portadoras de dor pélvica, a discussão principal é sobre a retirada total da pseudocápsula ou sua drenagem e coagulação. Num estudo prospectivo e comparativo entre estas duas técnicas laparoscópicas, mostrou-se que a recidiva de dor pélvica e o aparecimento de novos endometriomas foram mais elevados no grupo submetido a drenagem e coagulação da pseudocápsula, com taxas de complicação cirúrgica semelhantes ${ }^{20}$. Estes achados foram corroborados por outros, mostrando uma tendência na literatura por esta técnica, que também permite obter material para análise anatomopatológica ${ }^{76}$. Jones et al. (2002) $)^{77}$ publicaram trabalho comparativo mostrando uma tendência dos ginecologistas no Reino Unido para realizar somente drenagem e coagulação dos endometriomas, com iguais taxas de recidiva na dor pélvica.

Nos casos em que há endometriose infiltrativa profunda, seja ela de septo reto-vaginal, intestinal ou vesical, o benefício do tratamento cirúrgico é muito grande. A laparoscopia pode ser realizada com sucesso para abordagem da endometriose do septo reto-vaginal, proporcionando grande melhora na dor pélvica crônica e cursando com baixas taxas de complicação ${ }^{78}$. A via laparoscópica também foi utilizada com grande sucesso para ressecção de endometriose infiltrativa profunda acometendo o retosigmóide. Estes autores realizaram a ressecção segmentar via vaginal do reto-sigmóide, infiltrado por endometriose, assistida por laparoscopia ${ }^{79}$. Com isso, podemos concluir que a melhora clínica dos sintomas ginecológicos e intestinais que acometem estas mulheres é significativa com o tratamento cirúrgico, que deve ser sempre encorajado, porém realizado por cirurgião experiente, e a paciente deve estar ciente das possiveis complicações ${ }^{80}$.

\section{Conclusões}

A prevalência da endometriose é bastante elevada, especialmente em pacientes portadoras de infertilidade e dor pélvica crônica. O impacto bio-psico-social desta doença intrigante e enigmática é elevado, tanto em nivel individual, como de saúde pública. O tratamento sempre deve ser individualizado, considerando não só as evidências existentes em relação à eficácia dos diferentes regimes terapêuticos, como as demais variáveis determinantes do sucesso terapêutico. Em última instância, deve-se buscar a promoção da melhoria global na qualidade de vida das pacientes. É importante ressaltar que a controvérsia sobre o tratamento da endometriose e seus sintomas são inúmeras, especialmente aquelas relacionadas com infertilidade associada a esta doença. Portanto, as recomendações resumidas a seguir poderão ser revistas à medida que estudos clínicos randomizados, controlados e com casuística adequada gerarem evidências mais concretas e confiáveis.

Em pacientes com endometriose mínima ou leve, a supressão da função ovariana não é efetiva na melhora da fertilidade, mas a ablação das lesões, associada à adesiólise, parece ser mais efetiva do que a realização exclusiva da laparoscopia diagnóstica. Conduta expectante ou estimulação ovariana associada à inseminação intra-uterina ou FIVETE podem ser consideradas, dependendo de outros fatores como idade da paciente, tempo de infertilidade, presença de fator masculino associado e resposta a terapias prévias.

Não dispomos de evidências suficientes para determinar se a excisão cirúrgica, em caso de doença moderada ou severa, melhoraria as taxas de gestação.

A FIV parece ser uma abordagem adequada, especialmente nos casos em que há coexistência de fatores de infertilidade e/ou falha de outros tratamentos. Quando a realização de FIV é indicada, recomenda-se avaliar a possibilidade de usar 
agonistas do GnRH por 3 a 6 meses, previamente à realização da estimulação ovariana controlada.

Verifica-se que a supressão da função ovariana por 3 a 6 meses em pacientes com doença confirmada reduz a dor associada à endometriose.

Todas as medicações estudadas (AMP, gestrinona, COC, danazol e GnRHa) mostram-se igualmente efetivas no alivio da dor. Contudo, os efeitos adversos apresentados e os custos são diferentes e devem ser considerados quando da escolha terapêutica.

A ablação das lesões endometrióticas reduz a dor associada a endometriose, sendo menos efetiva nos casos de doença mínima.

Dependendo da gravidade da doença encontrada, a prática ideal consiste em realizar diagnóstico e exérese cirúrgica dos focos de endometriose no mesmo ato operatório. Não há dados concretos que justifiquem a prescrição de tratamento hormonal antes da cirurgia, para melhorar as taxas de sucesso da abordagem cirúrgica.

O tratamento pós-operatório com danazol ou GnRHa por seis meses, após a cirurgia, reduz a dor associada a endometriose e posterga a recorrência da doença, quando comparado com placebo ou conduta expectante. Contudo, o tratamento pósoperatório com COC parece não ser tão efetivo.

A exérese de endometriomas com diâmetro $>4 \mathrm{~cm}$ parece melhorar a taxa de fecundidade natural e aquela após procedimentos de reprodução assistida, além de reduzir a dor e postergar a recidiva da doença.

\section{Referências}

1. Borges LS, Rosa e Silva JC, Rosa e Silva ACJS, Aguiar FM, Poli Neto OB, Reis FJC, et al. Avaliação da concordância diagnóstica entre métodos não invasivos e endoscopia na investigação de infertilidade. Rev Bras Ginecol Obstet. 2005;27(7):401-6.

2. Abrão MS, Podgaec S, Filho BM, Ramos LO, Pinotti JA, de Oliveira RM. The use of biochemical markers in the diagnosis of pelvic endometriosis. Hum Reprod. 1997;12(11):2523-7.

3. Low WY, Edelmann RJ, Sutton C. A psychological profile of endometriosis patients in comparison to patients with pelvic pain of other origins. J Psychosom Res. 1993;37(2):111-6.

4. Chandra A, Mosher WD. The demography of infertility and the use of medical care for infertility. Infertil Reprod Med Clin North Am. 1994;5(2):283-96.

5. Schwartz D, Mayaux MJ. Female fecundity as a function of age: results of artificial insemination in 2193 nulliparous women with azoozpermic husbands. Federation CECOS. N Engl J Med. 1982;306(7):404-6.
6. Marcoux S, Maheux R, Berube S. Laparoscopic surgery in infertile women with minimal or mild endometriosis. Canadian Collaborative Group on Endometriosis. N Engl J Med. 1997;337(4):217-22.

7. Parazzini F. Ablation of lesions or no treatment in minimal-mild endometriosis in infertile women: a randomized trial. Gruppo Italiano per lo Studio dell'Endometriosi. Hum Reprod. 1999;14(5):1332-4.

8. Hughes E, Fedorkow D, Collins J, Vandekerckhove P. Ovulation suppression for endometriosis. Cochrane Database Syst Rev. 2003;(3):CD000155.

9. Dicker D, Goldman GA, Ashkenazi J, Feldberg D, Voliovitz I, Goldman JA. The value of pre-treatment with long-term gonadotropin-releasing hormone (GnRH) analogue in IVF-ET therapy of severe endometriosis. Hum Reprod. 1990;5(4):418-20.

10.Marcus SF, Edwards RG. High rates of pregnancy after long term down regulation of women with severe endometriosis. Am J Obstet Gynecol. $1994 ; 171(3): 812-7$.

11.Surrey ES, Silverberg KM, Surrey MW, Schoolcraft WB. The effect of prolonged gonadotropin-releasing hormone agonist therapy on the outcome of in vitro fertilization-embryo transfer in patients with endometriosis. Fertil Steril. 2002;78(4):699-704.

12.Sallan HN, Garcia-Velasco JA, Dias S, Arici A. Long-term pituitary down-regulation before in vitro fertilization (IVF) for women with endometriosis. Cochrane Database Syst Rev. 2006;25(1):CD004635.

13.Allaire C. Endometriosis and infertility: a review. J Reprod Med. 2006;51(3):164-8.

14.Jacobson TZ, Barlow DH, Koninckx PR, Olive D, Farquhar C. Laparoscopic surgery for subfertility associated with endometriosis. Cochrane Database Syst Rev. 2002;(4):CD001398.

15.Adamson GD, Hurd SJ, Pasta DJ, Rodriguez BD. Laparoscopic endometriosis treatment: is it better? Fertil Steril. 1993;59(1):35-44.

16.Guzick DS, Silliman NP, Adamson GD, Buttram VC $\mathrm{Jr}$, Canis M, Malinak LR, et al. Prediction of pregnancy in infertile women based on the American Society for Reproductive Medicine's revised classification of endometriosis. Fertil Steril 1997;67(5):822-9.

17. Osuga Y, Koga K, Tsutsumi O, Yano T, Maruyama M, Kugu K, et al. Role of laparoscopy in the treatment of endometriosis-associated infertility. Gynecol Obstet Invest. 2002;53 Suppl 1:33-9.

18. Kennedy S, Bergqvist A, Chapron C, D'Hooghe T, Dunselman G, Greb R, et al. ESHRE guideline for the diagnosis and treatment of endometriosis. Hum Reprod. 2005;20(10):2698-704.

19. Hart R, Hickey M, Maouris P, Buckett W, Garry R. Excisional surgery versus ablative surgery for ovarian endometriomata: a Cochrane Review. Hum Reprod. 2005;20(11):3000-7. 
20.Beretta P, Franchi M, Ghezzi F, Busacca M, Zupi E, Bolis P. Randomized clinical trial of two laparoscopic treatments of endometriomas: cystectomy versus drainage and coagulation. Fertil Steril. 1998;70(6):1176-80.

21.Chapron C, Vercellini P, Barakat H, Vieira M, Dubuisson JB. Management of ovarian endometriomas. Hum Reprod Update. 2002;8(6):591-7.

22.Vercellini P, Chapron C, De Giorgi O, Consonni D, Frontino G, Crosignani PG. Coagulation or excision of ovarian endometriomas? Am J Obstet Gynecol. 2003;188(3):606-10.

23.Nargund G, Cheng WC, Parsons J. The impact of ovarian cystectomy on ovarian response to stimulation during in-vitro fertilization cycles. Hum Reprod. 1996;11(1):81-3.

24.Loh FH, Tan AT, Kumar J, Ng SC. Ovarian response after laparoscopic ovarian cystectomy for endometriotic cysts in 132 monitored cycles. Fertil Steril. 1999;72(2):316-21.

25.Hachisuga T, Kawarabayashi T. Histopathological analysis of laparoscopically treated ovarian endometriotic cysts with special reference to loss of follicles. Hum Reprod. 2002;17(2):432-5.

26.Geber S, Ferreira DP, Spyer Prates LF, Sales L, Sampaio M. Effects of previous ovarian surgery for endometriosis on the outcome of assisted reproduction treatment. Reprod Biomed Online. 2002;5(2):162-6.

27. Ho HY, Lee RK, Hwu YM, Lin MH, Su JT, Tsai YC. Poor response of ovaries with endometrioma previously treated with cystectomy to controlled ovarian hyperstimulation. J Assist Reprod Genet. 2002;19(11):507-11.

28. Somigliana E, Ragni G, Benedetti F, Borroni R, Vegetti W, Crosignani PG. Does laparoscopic excision of endometriotic ovarian cysts significantly affect ovarian reserve? Insights from IVF cycles. Hum Reprod. 2003;18(11):2450-3.

29.Bianchi S, Busacca M, Agnoli B, Candiani M, Calia C, Vignali M. Effects of 3 month therapy with danazol after laparoscopic surgery for stage III/IV endometriosis: a randomized study. Hum Reprod. 1999;14(5):1335-7.

30.Busacca M, Somigliana E, Bianchi S, De Marinis S, Calia C, Candiani M, et al. Post-operative GnRH analogue treatment after conservative surgery for symptomatic endometriosis stage III-IV: a randomized controlled trial. Hum Reprod. 2001;16(11):2399-402.

31.Vercellini P, Crosignani PG, Fadini R, Radici E, Belloni C, Sismondi P. A gonadotrophin-releasing hormone agonist compared with expectant management after conservative surgery for symptomatic endometriosis. Br J Obstet Gynaecol. 1999:106(7):672-7.

32. Chaffkin LM, Nulsen JC, Luciano AA, Metzger DA. A comparative analysis of the cycle fecundity rates associated with combined human menopausal gonadotrophin (hMG) and intrauterine insemination
(IUI) versus either hMG or IUI alone. Fertil Steril. 1991;55(2):252-7.

33.Deaton JL, Gibson M, Blackmer KM, Nakajima ST, Badger GJ, Brumsted JR. A randomized, controlled trial of clomiphene citrate and intrauterine insemination in couples with unexplained infertility or surgically corrected endometriosis. Fertil Steril. 1990;54(6):1083-8.

34.Barnhart K, Dunsmoor-Su R, Coutifaris C. Effect of endometriosis on in vitro fertilization. Fertil Steril. 2002;77(6):1148-55.

35.Kistner RW. The use of newer progestins in the treatment of endometriosis. Am J Obstet Gynecol. 1958;75(2):264-78.

36. Luciano AA, Turksoy RN, Carleo J. Evaluation of oral medroxyprogesterone acetate in the treatment of endometriosis. Obstet Gynecol. 1988;72(3 Pt 1):323-7.

37.Harrison RF, Barry-Kinsella C. Efficacy of medroxyprogesterone treatment in infertile women with endometriosis: a prospective, randomized, placebocontrolled study. Fertil Steril. 2000;74(1):24-30.

38.Vercellini P, De Giorgi O, Oldani S, Cortesi I, Panazza $\mathrm{S}$, Crosignani P. Depot medroxyprogesterone acetate versus an oral contraceptive combined with very low-dose danazol for long-term treatment of pelvic pain associated with endometriosis. Am J Obstet Gynecol. 1996;175(2):396-401.

39. Nieto A, Tacuri C, Serra M, Keller J, Cortes-Prieto J. Long-term follow-up of endometriosis after two different therapies (gestrinone and buserelin). Clin Exp Obstet Gynecol. 1996;23(4):198-204.

40.Gestrinone versus a gonadotropin-releasing hormone agonist for the treatment of pelvic pain associa-ted with endometriosis: a multicenter, randomized, double-blind study. Gestrinone Italian Study Group. Fertil Steril. 1996;66(6):911-9.

41.Fedele L, Arcaini L, Bianchi S, Baglioni A, Vercellini P. Comparison of cyproterone acetate and danazol in the treatment of pelvic pain associated with endometriosis. Obstet Gynecol. 1989;73(6):1000-4.

42.Muneyyirci-Delale O, Karacan M. Effect of norethindrone acetate in the treatment of symptomatic endometriosis. Int J Fertil Womens Med.1998; 43(1):24-7.

43.Yisa SB, Okenwa AA, Husemeyer RP. Treatment of pelvic endometriosis with etonogestrel subdermal implant (Implanon). J Fam Plann Reprod Health Care. 2005;31(1):67-70.

44.Vercellini P, Aimi G, Panazza S, De Giorgi O, Pesole A, Crosignani PG. A levonorgestrel-releasing intrauterine system for the treatment of dysmenorrhea associated with endometriosis: a pilot study. Fertil Steril. 1999;72(3):505-8.

45. Fedele L, Bianchi S, Zanconato G, Portuese A, Raffaelli $\mathrm{R}$. Use of levonorgestrel-releasing intrauterine device 
in the treatment of rectovaginal endometriosis. Fertil Steril. 2001;75(3):485-8.

46.Petta CA, Ferriani RA, Abrao MS, Hassan D, Rosa e Silva JC, Podgaec S, et al. Randomized clinical trial of a levonorgestrel-releasing intrauterine system and a depot GnRH analogue for the treatment of chronic pelvic pain in women with endometriosis. Hum Reprod. 2005;20(7):1993-8.

47. Rosa e Silva AC, Rosa e Silva JC, Nogueira AA, Petta CA, Abrao MS, Ferriani RA. The levonorgestrel-releasing intrauterine device reduces CA-125 serum levels in patients with endometriosis. Fertil Steril. 2006; 86(3)742-4.

48. Vercellini P, Trespidi L, Colombo A, Vendola N, Marchini M, Crosignani P. A gonadotropin-releasing hormone agonist versus a low-dose oral contraceptive for pelvic pain associated with endometriosis. Fertil Steril. 1993;60(1):75-9.

49.Muzii L, Marana R, Caruana P, Catalano GF, Margutti F, Panici PB. Postoperative administration of monophasic combined oral contraceptives after laparoscopic treatment of ovarian endometriomas: a prospective, randomized trial. Am J Obstet Gynecol. 2000;183(3):588-92.

50.Edelman AB, Gallo MF, Jensen JT, Nichols MD, Schulz KF, Grimes DA. Continuous or extended cycle vs. cyclic use of combined oral contraceptives for contraception. Cochrane Database Syst Rev. 2005;(3):CD004695.

51.Greenblatt R, Dmowski W, Mahesh VB, Scholer HF. Clinical studies with an antigonadotropin-danazol. Fertil Steril. 1971;22(2):102-12.

52.Barbieri RL, Evans S, Kistner RW. Danazol in the treatment of endometriosis: analysis of 100 cases with a 4-year follow-up. Fertil Steril. 1982; 37(6):737-46.

53. Henzl MR, Corson SL, Moghissi K, Buttram VC, Berqvist C, Jacobson J. Administration of nasal nafarelin as compared with oral danazol for endometriosis. A multicenter double-blind comparative clinical trial. N Engl J Med. 1988;318(8):485-9.

54.Cirkel U, Ochs H, Schneider HP. A randomized, comparative trial of triptorelin depot (D-Trp6-LHRH) and danazol in the treatment of endometriosis. Eur J Obstet Gynecol Reprod Biol. 1995;59(1):61-9.

55.Biberoglu KO, Behrman SJ. Dosage aspects of danazol therapy in endometriosis: short-term and long-term effectiveness. Am J Obstet Gynecol. $1981 ; 139(6): 645-54$

56.Meldrum DR, Chang RJ, Lu J, Vale W, Rivier J, Judd H. "Medical oophorectomy" using a long-acting GnRH agonist: a possible new approach to the treatment of endometriosis. J Clin Endocrinol Metab. 1982;54(5):1081-3.

57.Dlugi AM, Miller JD, Knittle J. Lupron depot (leuprolide acetate for depot suspension) in the treatment of endometriosis: a randomized, placebo-controlled, double-blind study. Lupron Study Group. Fertil Steril. 1990;54(3):419-27.

58.Ling FW. Randomized controlled trial of depot leuprolide in patients with chronic pelvic pain and clinically suspected endometriosis. Pelvic Pain Study Group. Obstet Gynecol. 1999;93(1):51-8.

59.Hornstein MD, Hemmings R, Yuzpe AA, Heinrichs WL. Use of nafarelin versus placebo after reductive laparoscopic surgery for endometriosis. Fertil Steril. 1997;68(5):860-4.

60.Jacobson J, Harris SR, Bullingham RE. Low dose intranasal nafarelin for the treatment of endometriosis. Acta Obstet Gynecol Scand. 1994;73(2):144-50.

61.Fedele L, Bianchi S, Bocciolone L, Di Nola G, Franchi D. Buserelin acetate in the treatment of pelvic pain associated with minimal and mild endometriosis: a controlled study. Fertil Steril. 1993;59(3): 516-21.

62.Hornstein M, Yuzpe AA, Burry KA, Heinrichs LR, Buttram VL Jr, Orwoll ES. Prospective randomized double-blind trial of 3 versus 6 months of nafarelin therapy for endometriosis associated pelvic pain. Fertil Steril. 1995;63(5):955-62.

63.Parazzini F, Fedele L, Busacca M, Falsetti L, Pellegrini $\mathrm{S}$, Venturini PL, et al. Postsurgical medical treatment of advanced endometriosis: results of a randomized clinical trial. Am J Obstet Gynecol. 1994;171(5): 1205-7.

64.Henzl MR, Kwei L. Efficacy and safety of nafarelin in the treatment of endometriosis. Am J Obstet Gynecol. 1990;162(2):570-4.

65. Scialli AR, Jestila KJ, Simon JA. Leuprolide acetate and bone mineral density measured by quantitative digitized radiography. Fertil Steril. 1993;59(3):674-6.

66. Mukherjee T, Barad D, Turk R, Freeman R. A randomized, placebo-controlled study on the effect of cyclic intermittent etidronate therapy on the bone mineral density changes associated with six months of gonadotropin-releasing hormone agonist treatment. Am J Obstet Gynecol. 1996;175(1):105-9.

67. Surrey ES. Add-back therapy and gonadotropinreleasing hormone agonists in the treatment of patients with endometriosis: can a consensus be reached? Add-Back Consensus Working Group. Fertil Steril. 1999;71(3):420-4.

68. Clayton RD, Hawe JA, Love JC, Wilkinson N, Garry R. Recurrent pain after hysterectomy and bilateral salpingo-oophorectomy for endometriosis: evaluation of laparoscopic excision of residual endometriosis. $\mathrm{Br}$ J Obstet Gynaecol. 1999;106(7):740-4.

69. Crosignani PG, Vercellini P, Biffignandi F, Costantini W, Cortesi I, Imparato E. Laparoscopy versus laparotomy in conservative surgical treatment for severe endometriosis. Fertil Steril. 1996;66(5):706-11. 
70.Busacca M, Fedele L, Bianchi S, Candiani M, Agnoli B, Raffaelli R, et al. Surgical treatment of recurrent endometriosis: laparotomy versus laparoscopy. Hum Reprod. 1998;13(8):2271-4.

71.Catalano GF, Marana R, Caruana P, Muzii L, Mancuso S. Laparoscopy versus microsurgery by laparotomy for excision of ovarian cysts in patients with moderate or severe endometriosis. J Am Assoc Gynecol Laparosc. 1996;3(2):267-70.

72.Ekstein P, Szold A, Sagie B, Werbin N, Klausner $\mathrm{JM}$, Weinbroum AA. Laparoscopic surgery may be associated with severe pain and high analgesia requirements in the immediate postoperative period. Ann Surg. 2006:243(1):41-6.

73. Wright J, Lotfallah H, Jones K, Lovell D. A randomized trial of excision versus ablation for mild endometriosis. Fertil Steril. 2005;83(6):1830-6.

74. Sutton CJ, Pooley AS, Ewen SP, Haines P. Followup report on a randomized controlled trial of laser laparoscopy in the treatment of pelvic pain associated with minimal to moderate endometriosis. Fertil Steril. 1997;68(6):1070-4.

75.Abbott J, Hawe J, Hunter D, Holmes M, Finn P, Garry R. Laparoscopic excision of endometriosis: a randomized, placebo-controlled trial. Fertil Steril. 2004;82(4):878- 84 .

76.Saleh A, Tulandi T. Reoperation after laparoscopic treatment for ovarian endometriomas by excision and by fenestration. Fertil Steril. 1999;72(2): 322-4.

77. Jones KD, Fan A, Sutton CJ. The ovarian endometrioma: why is it so poorly managed? Indicators from an anonymous survey. Hum Reprod. 2002;17 (4):845-9.

78. Chapron C, Jacob S, Dubuisson JB, Vieira M, Liaras E, Fauconnier A. Laparoscopically assisted vaginal management of deep endometriosis infiltrating the rectovaginal septum. Acta Obstet Gynecol Scand. $2001 ; 80(4): 349-54$.

79.Abrao MS, Sagae UE, Gonzales M, Podgaec $\mathrm{S}$, Dias JA Jr. Treatment of rectosigmoid endometriosis by laparoscopically assisted vaginal rectosigmoidectomy. Int J Gynaecol Obstet. 2005; 91(1):27-31.

80.Dubernard G, Piketty M, Rouzier R, Houry S, Bazot M, Darai E. Quality of life after laparoscopic colorectal resection for endometriosis. Hum Reprod. 2006;21(5):1243-7. 